\title{
The post-buckling response of stiffened CFRP panels using a single-stringer compression specimen (SSCS): a numerical investigation
}

\author{
M. Ali Sadiq \& H. Qing \\ School of Aeronautics, Northwestern Polytechnical University, China
}

\begin{abstract}
The safe exploitation of the post-buckling region of CFRP panels in aerospace applications is of prime interest here. Due to the long computational time of finite element analysis for the post-buckling response of CFRP panels, there is a need to devise some efficient procedures. Skin/stringer debonding is primarily responsible for the collapse of the stiffened panels under compressive loading. In this study a simplified approach based on a single-stringer compression specimen (SSCS) has been employed to gain an insight into the post-buckling behaviour of CFRP panels under axial compression. A progressive damage model is used to get more accurate numerical results of the post-buckling response of SSCS. The outcomes of the study are compared with the published results and a fair agreement is found. In addition, a comparative study of open- and closed-section stringer stiffened panels based on a global-local approach for predicting pre-buckling, post-buckling and mode shapes is carried out. As the simplified model is used in this study so detailed damage models can be constructed to account for all damage modes: matrix cracking, fibre kinking, fibre fracture, and delamination. The study can be employed as a global-local approach to get the post-buckling response of CFRP stiffened panels. Furthermore, it also provides an efficient mean for the design optimization of CFRP structures in the post-buckling regime.
\end{abstract}

Keywords: CFRP, post-buckling, stiffened panels, SSCS, progressive damage, global-local approach.

\section{Introduction}

The interest for primary composite structures in new aircraft is growing, as evidenced by the Boeing 787, Airbus A350, and other smaller regional aircraft. These composite structures often consist of stiffened thin-walled constructions 
that can buckle under compressive or shear loads. Consequently, if these structures are to meet the highest safety and performance expectations, their post-buckling response and failure modes must be well understood.

Since stiffened curved panels are by far the most consumed structural component, it is important to study the behaviour of structural degradation to evaluate the safe design guidelines. Current numerical procedures cannot be directly applied for optimization of the post-buckling behaviour of stiffened cylindrical composite structures with sufficient reliability and efficiency. Thus investigation of stiffened composite cylindrical shells under axial load in their pre-buckling and post-buckling state is essential. One way to improve over-conservative designs is to produce a more reliable determination of collapse models in the post-buckling region like reduction of stiffness due to fibre over matrix failure.

The instability of laminated structures is one of the most complex problems in structural mechanics, and a series of research work has been carried out in the past decades. A pre-research project POSICOSS (Improved Post-buckling Simulation for Design of Fibre Composite Stiffened Fuselage Structures) started in Europe since 2000 [1], with main purpose to develop fast and reliable procedures for post-buckling analysis of fibre composite airframe structures, create experimental data base, and propose design guidelines. Another project COCOMAT (Improved Material Exploitation at Safe Design of Composite Airframe Structures by Accurate Simulation of Collapse) [2], was carried out to explore the accurate and reliable analysis methods of post-buckling up to collapse, and a series of simulation principles for buckling behaviours were investigated.

Furthermore, numerous experimentations on stiffened panels [3-5] and closed form configurations [6] had been conducted to get an insight into the complex post-buckling phenomenon. The high cost of manufacturing prototype composite structures and the difficulty to set up high fidelity tests with a complete set of measurements, make these tests quite complex and expensive to execute.

In recent years, several strategies have been developed for the study of the damage onset and propagation, including skin/stringer delamination. A finite element analysis tool for design and certification of aerospace structures is presented by Orifici et al. [7], which incorporates a global-local analysis technique for predicting interlaminar damage initiation, and degradation models to capture the growth of a pre-existing interlaminar damage region, such as a delamination or skin-stiffener debond, and in-plane ply damage mechanisms such as fibre fracture and matrix cracking.

Despite their efficiency, analytical and semi-analytical methods can be developed only by introducing a number of simplifying assumptions. In general, the failure of post-buckled panels involves several nonlinear mechanisms, including geometric as well as material nonlinearities, which cannot be captured properly by analytical models. Finally, the inadequacy of analytical models can be exacerbated by the existence of different failure mechanisms, including interlaminar and intralaminar damage phenomena.

More Recently finite element models with three levels of approximation have been developed by Vescovini et al. [8-10]. The first model is based on a relatively 
coarse mesh to capture the global post-buckling response of a multi-stringer panel. The second model can predict the nonlinear response as well as the debonding and crippling failure mechanisms in a single-stringer compression specimen (SSCS). The third model consists of a simplified version of the SSCS that is designed to minimize the computational effort. Simplified and computationally efficient models are developed that can predict the response and collapse of a stiffened panel. Cohesive elements are used to capture skin/stiffener separation at three levels of structural approximation. The result is a set of simplified models that are sufficiently fast for conducting sensitivity studies and sufficiently accurate to provide deeper understanding about the phenomena that lead to local and global collapse. Inexpensive to manufacture SSCS specimens are used to investigate the post-buckling response and the collapse with a co-cured hat stringer by Bisagni and Dávila [11]. Test specimens can be useful for the evaluation of damage tolerance of post-buckled structures and could therefore fill the gap between test coupons and multi-stringer panels in the building block approach to the design and certification of aerospace structures.

The dual objective of the present work is to use the progressive damage model to get the accurate numerical results for the post-buckling response of SSC specimens that are representative of the response of stiffened panels. In addition by using this model comparative study of open- and closed-section stringer stiffened CFRP panels is carried out.

\section{Analysis approach}

Different analysis tools such as progressive failure methods, continuum damage models, virtual crack closure technique, cohesive elements and X-FEM are available to study different aspects of the failure process. However different simplified approaches are used to reduce the computational costs associated with failure analysis of composite structures.

\subsection{Progressive damage}

A progressive damage model proposed by Alfano and Crisfield [14] has been used for the structural degradation. The damage initiation and propagation in fibrereinforced composites can be simulated with a nonlinear solution process. Damage activation functions based on the LaRC04 failure criteria [13] are used to predict the different failure mechanisms occurring at the ply level.

A bilinear cohesive zone model (CZM) [14] is used to simulate Interface delamination of adhesive joint between skin and stringer. Debonding is complete when eqn. (1) is satisfied.

$$
\left(\frac{G_{n}}{G_{c n}}\right)+\left(\frac{G_{t}}{G_{c t}}\right)=1
$$

where,

$\mathrm{G}_{\mathrm{n}}, \mathrm{G}_{\mathrm{t}}=$ Normal and tangential energy release rates respectively.

$\mathrm{G}_{\mathrm{cn}}, \mathrm{G}_{\mathrm{ct}}=$ Normal and tangential critical energy release rates respectively. 


\section{Numerical analysis}

The analyses in the following sections are conducted using ANSYS implicit solution procedure. The models use four noded shell 181 elements. For interface between skin and stringer flanges target 170 and contact 173 elements are used with default contact stiffness. Both the skin and the stringers are made from IM7/8552 graphite-epoxy material. The material's elastic, interlaminar, strength and fracture toughness properties [8] are summarized in Tables 1, 2, 3 and 4 respectively. The in-situ ply strengths [13], which account for the thickness of the ply and its position in the laminate (inner or outer ply), are ignored.

Table 1: Engineering Properties - IM7/8552.

\begin{tabular}{|c|c|c|c|c|c|}
\hline $\begin{array}{c}\mathrm{E}_{11} \\
(\mathrm{MPa})\end{array}$ & $\begin{array}{c}\mathrm{E}_{22} \\
(\mathrm{MPa})\end{array}$ & $\mathrm{G}_{12}(\mathrm{MPa})$ & $v_{12}$ & $\alpha_{1}\left(10^{-6 \circ} c^{-1}\right)$ & $\alpha_{2}\left(10^{-6 \circ} c^{-1}\right)$ \\
\hline 150,000 & 9080 & 5290 & 0.32 & -5.5 & 25.8 \\
\hline
\end{tabular}

Table 2: Interlaminar Properties - IM7/8552.

\begin{tabular}{|c|c|c|c|}
\hline $\mathrm{GI}_{\mathrm{c}}(\mathrm{N} / \mathrm{mm})$ & $\mathrm{GII}_{\mathrm{c}}(\mathrm{N} / \mathrm{mm})$ & $\sigma_{z Z}(\mathrm{MPa})$ & $\sigma_{x z}(\mathrm{MPa})$ \\
\hline 0.277 & 0.788 & 50 & 100 \\
\hline
\end{tabular}

Table 3: Ply Strength Properties, MPa - IM7/8552.

\begin{tabular}{|c|c|c|c|c|}
\hline $\mathrm{XT}$ & $\mathrm{XC}$ & $\mathrm{YT}$ & $\mathrm{YC}$ & $\mathrm{SL}$ \\
\hline 2323 & 1200 & 160.2 & 199.8 & 130.2 \\
\hline
\end{tabular}

Table 4: Fracture Toughness Properties N/mm - IM7/8552.

\begin{tabular}{|c|c|c|c|}
\hline $\mathrm{GF}(+)$ & GF(-) & GMI & GMII \\
\hline 81.5 & 106.3 & 0.277 & 0.788 \\
\hline
\end{tabular}

In the case of multi-stringer panels, the complexity of the interaction between post-buckling and damage renders their prediction computationally impractical. Therefore, by identifying repeating features that characterize the response of a multi-stringer panel, it is possible to determine the dimensions of a single-stringer specimen whose response is similar to that of the multi-stringer panel in terms of post-buckling deformation failure mechanisms.

The multi-stringer panel under investigation is composed of five stringers. Panel has total length of $720 \mathrm{~mm}$ and a width of $680 \mathrm{~mm}$. The height of the hat stringer is $30 \mathrm{~mm}$; the width of the crown top is $15 \mathrm{~mm}$, while the web is at an angle of $25^{\circ}$ with the normal to the skin. The skin consists of 8-ply quasi-isotropic laminate with stacking sequence of $\left[45^{\circ} / 90^{\circ} /-45^{\circ} / 0^{\circ}\right]_{\mathrm{s}}$. The stringers are composed of 7-ply laminate with a symmetric stacking sequence of $\left[-45^{\circ} / 0^{\circ} / 45^{\circ} / 0^{\circ} / 45^{\circ} / 0^{\circ} /-45^{\circ}\right]$. 


\subsection{Hat stiffened single-stringer compression specimen (SSCS)}

A single-stringer compression specimen (SSCS) is composed of one stringer, and extends transversally from half bay to half bay, for a total width of $150 \mathrm{~mm}$. Through parametric studies, it was found that a SSC specimen length of $240 \mathrm{~mm}$ resulted in a stress distribution that is similar to that observed in the multi-stringer panel [8].

Progressive damage model available with ANSYS 14.0 is used. Damage initiation (DMGI) based on LaRc04 failure criteria is defined. Material damage evolution law (DMGE) following the initiation of damage instant stiffness reduction. The skin-stringer separation failure is modelled by placing a layer of cohesive elements between the skin and the stringer along the flanges of the stringer. These elements can represent mixed-mode delamination growth with a damage evolution law specified as a bilinear constitutive equation.

A reduction of the interlaminar strength determines an increase of the length of the process zone [9]. Therefore, the local model uses a typical mesh size of $1.0 \times 5.0 \mathrm{~mm}$ (flange width $\times$ flange length direction) for interface elements with reduction of interlaminar strength of $50 \mathrm{MPa}$ to $25 \mathrm{MPa}$. The periodic symmetric boundary conditions at the free edges of SSCS are employed.

Fig. 1 reports a comparison of load-displacement curves obtained for a typical hat-stiffened SSCS test specimen, Abaqus results [8] and the present study, which shows the validity of present model.

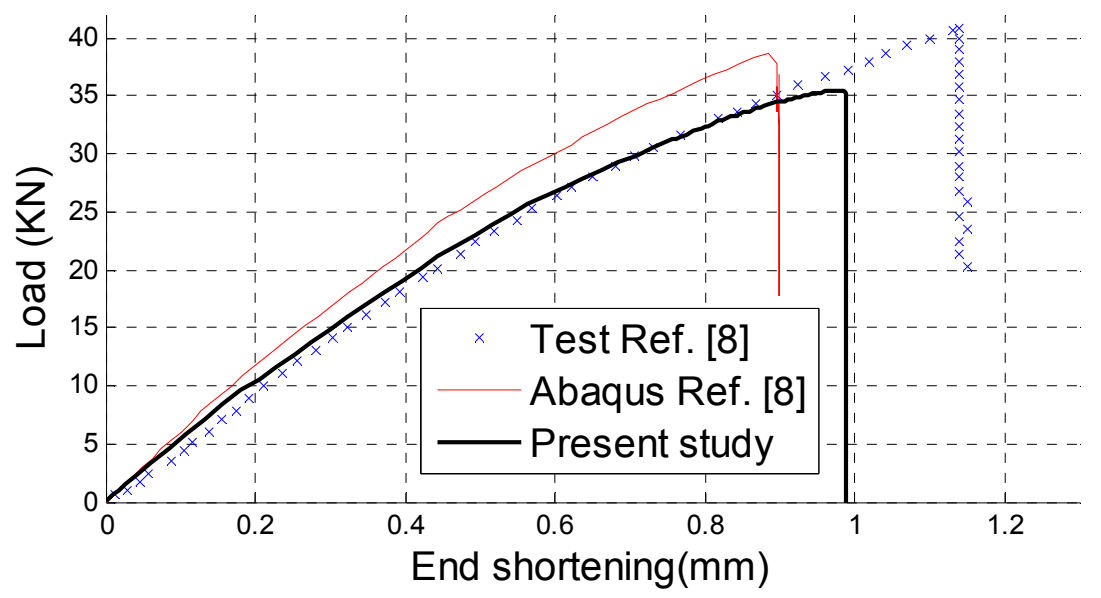

Figure 1: Numerical and experimental force-displacement curves of hat-stiffened SSCS.

The results indicate that the load displacement responses based on the progressive damage model are comparable with the published experimental results. 


\section{Comparison of hat- and T-stiffened panels}

When complex loading or structural configuration must be considered the assumption of periodicity becomes invalid, so the response of the panel must be conducted on full multi-stringer panel rather than on a smaller specimen. In order to identify critical areas of multi-stringer panels, a computationally efficient global-local approach is used.

\subsection{Global model/ multi-stringer panels}

The goal of the global analysis step is to determine the displacement field of the entire multi-stringer panel. Using the global-local approach the analysis of the entire panel is performed using a model with a relatively coarse mesh.

Global model is composed exclusively of shell elements and is therefore unable to capture the onset and the propagation of delaminations. In addition, the material response is assumed to be linear elastic. Therefore, no convergence difficulties occur during the quasi static load incrementation.

Two types of stiffened panels are modelled i.e. $\mathrm{T}$ and hat. The overall dimensions of both the panels are same as mentioned in section 3.1. The panel is stiffened longitudinally by five stringers. The T stringer flange is $60 \mathrm{~mm}$ and web height is $25 \mathrm{~mm}$, adjusted in order to have the same panel weights.

Element size of $5 \mathrm{~mm}$ is selected along both longitudinal and lateral directions. A comparison of force-end shortening plot for hat and $\mathrm{T}$ stringer stiffened multistringer panels is shown in fig. 2. The out-of-plane deflection contours on the panels of global models for both the panels are shown in figs 3(a) and 3(b).

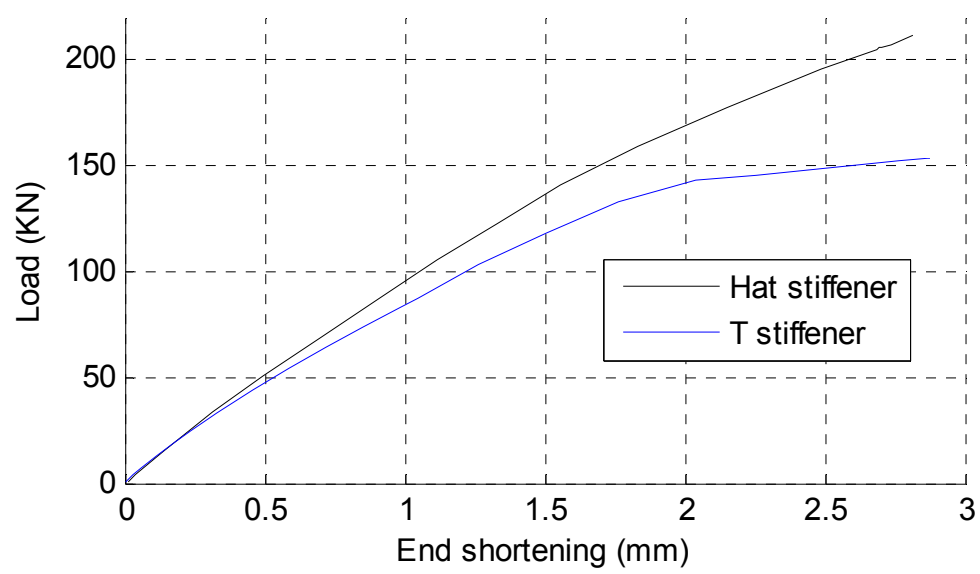

Figure 2: Comparison of force-end displacement curves of hat- and T-stiffened multi-stringer panels. 


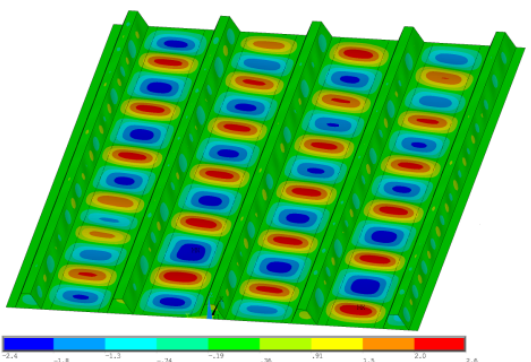

(a)

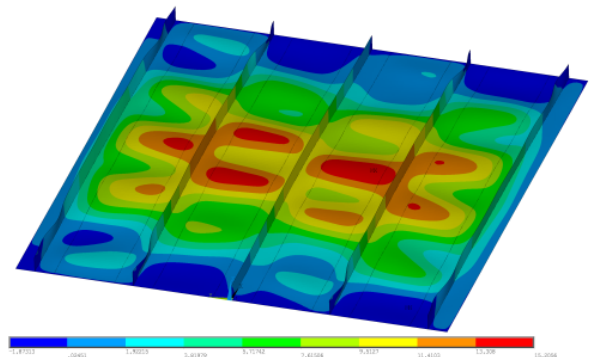

(b)

Figure 3: Out of plane displacements of (a) hat- and (b) T-stiffened multi-stringer panels.

The results of the multi-stringer panels show a local out of plane displacement for hat-stiffened stringer panel which leads to the local collapse of the CFRP panels. Whereas, for the T stringer stiffened panels the out of plane displacement shows a global response, which leads to the global collapse of the CFRP panels.

\subsection{Hat and T SSC specimens}

The same model proposed in section 3.1 is used here. Fig. 4(a) shows a comparison of load-displacement curves obtained for typical hat- and T-stiffened singlestringer compression specimens (SSCS). Figs 4(b) and 4(c) shows the out of plane displacement plots of hat- and T-stiffened SSC specimens respectively.

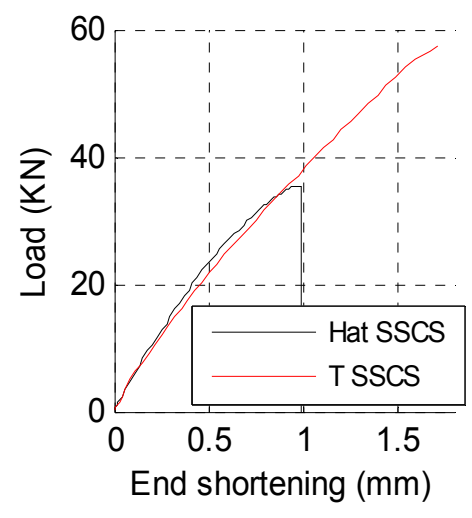

(a)

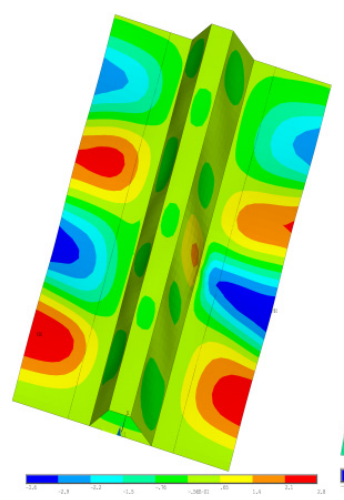

(b)

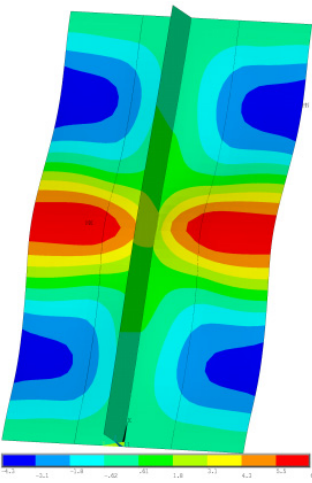

(c)

Figure 4: Comparison of compression response of hat- and T-stiffened panels (a) force-displacement curves (b) out of plane deformation of hat-stiffened panel (b) out of plane deformation of T-stiffened panel. 
Figs 5(a) and 5(b) shows the skin/stringer delamination zone of the hat- and Tstiffened SSCS respectively. Here the contours of the cohesive elements damage variables are shown. For hat stringer stiffened panel the delamination starts from inside the stringer flange due to the mode shift.

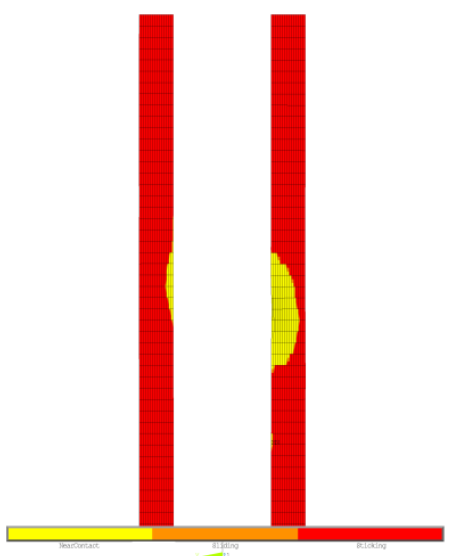

(a)

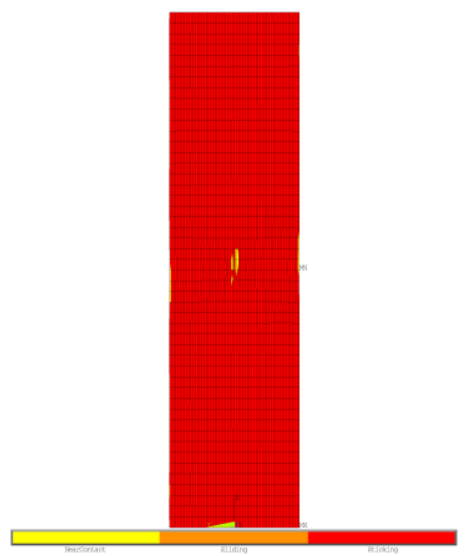

(b)

Figure 5: Delamination plot of (a) h and (b) T-stiffener contact zone.

\section{Results discussion}

From both global and the local model analysis results it is observed that $\mathrm{T}$ stringer stiffened panel showed the global out of plane deformation whereas, the hat stringer stiffened panel showed local out of plane deformation. It is also observed that the stiffness loss in case of $T$ stringer stiffened panel is more than corresponding hat stringer stiffened panel before the final collapse. Moreover, $\mathrm{T}$ stringer stiffened panel shows global failure mode which is more catastrophic.

For hat stringer stiffened panel, skin stringer delamination is the main cause of the failure and in case of $\mathrm{T}$ stringer stiffened panel, skin stringers debond start at higher load than the corresponding hat stringer stiffened panels.

Furthermore the buckling of the open-section stiffeners such as T-section stringers generally induces a loss of stiffness that may lead the stringer to twist and bend, which can trigger a global buckling mode.

\section{Conclusions}

An efficient parametric finite element model was developed to explore the postbuckling response of different types of stringer stiffened panels, as the post-buckling response is geometric driven. Firstly, a progressive damage model based analysis for hat-stiffened SSC specimen was conducted. The model is used 
to captures failure modes and a fair comparison with the published results was found. Therefore the progressive damage model based post-buckling analysis procedure presented in this study is capable to capture the failure modes. Secondly, a comparative study of post-buckling pattern for hat and $\mathrm{T}$ stringer stiffened panels was carried out.

The buckling of open-section stiffeners such as T-section stringers generally induces a loss of stiffness that may lead the stringer to twist and bend, which can trigger a global buckling mode, whereas, the hat stringer stiffened panels triggers the local buckling due to the skin stringer delamination. This is shown by the present study using more efficient global-local approach based on SSCS. So the model can be used for different type of stringer stiffened panels.

In addition, the understanding of the mechanism of collapse extracted from the analyses can also be used to develop an improved design decisions more efficiently.

\section{References}

[1] R. Zimmermann, R. Rolfes, POSICOSS-improved postbuckling simulation for design of fibre composite stiffened fuselage structures. Composite Structures 73(2), pp. 171-174, 2006.

[2] R. Degenhardt, R. Rolfes, R. Zimmermann, K. Rohwer, COCOMATimproved material exploitation of composite airframe structures by accurate simulation of postbuckling and collapse. 73(2), pp. 175-178, 2006.

[3] L. Lanzi, A numerical and experimental investigation on composite stiffened panels into post-buckling. Composite Structures 42, pp. 1645-1664, 2004.

[4] R. Zimmermann, H. Klein, A. Kling, Buckling and postbuckling of stringer stiffened fibre composite curved panels tests and computations. Composite Structures 73, pp. 150-161, 2006.

[5] D. Wilckens, F. Odermann, A. Kling, Buckling and Post Buckling of Stiffened CFRP Panels under Compression and Shear-Test and Numerical Analysis. 54th AIAA/ASME/ASCE/AHS/ASC Structures, Structural Dynamics, and Materials Conference Boston, Massachusetts, 2013.

[6] Abramovich H., Weller T., Bisagni C., Buckling Behaviour of Composite Laminated Stiffened Panels under Combined Shear-Axial Compression. Journal of Aircraft, 45(2), pp. 402-413, 2008.

[7] A. C. Orifici, R. S. Thomson, R. Degenhardt, J. Bayandor, Development of a finite element methodology for the collapse analysis of composite aerospace structures. Journal of Composite Materials 43: 3239, 2009.

[8] R. Vescovini, C. Bisagni, C.G. Dávila, Single-Stringer Compression Specimen for the Assessment of Damage Tolerance of Postbuckled Structures. Journal of Aircraft, 48(2), 2011.

[9] R. Vescovini, C.G. Dávila, C. Bisagni, Simplified Models for the Study of Postbuckled Hat-Stiffened Composite Panels. NASA/TM-2012-217336, 2012. 
[10] R. Vescovini, C.G. Dávila, C. Bisagni, Failure analysis of composite multistringer panels using simplified models .Composites: Part B, 45, pp. 939-951, 2013.

[11] C. Bisagni, C.G. Dávila, Experimental investigation of the postbuckling response and collapse of a single-stringer specimen. Composite Structures 108, pp. 493-503, 2014.

[12] F.K. Chang, K.Y. Chang, A Progressive Damage Model for Laminated Composites Containing Stress Concentrations. Journal of Composite Materials, 21, pp. 834-855, 1987.

[13] C.G. Davila, P.P. Camanho, C.A. Rose, Failure Criteria for FRP Laminates. Journal of Composite Materials, 39, pp. 323-345, 2005.

[14] G. Alfano, M.A. Crisfield, Finite element interface models for the delamination analysis of laminated composites: mechanical and computational issues. International Journal for Numerical Methods in Engineering, 50, pp. 1701-1736, 2001.

[15] F.K. Chang, M.H. Chen, Laminated Composites The In Situ Ply Shear Strength Distributions in Graphite/Epoxy. Journal of Composite Materials, 21, pp. 708-733, 1987. 